\title{
Ultrastructure of the Mesonotal Mycangium of Xylosandrus mutilatus (Blandford), an Exotic Ambrosia Beetle (Coleoptera: Curculionidae: Scolytinae) by Light, Scanning, and Transmission Electron Microscopy
}

\author{
W. D. Stone, ${ }^{*}$ T. E. Nebeker, ${ }^{*}$ and W. A. Monroe** \\ *Department of Entomology and Plant Pathology, **Electron Microscopy Center, Mississippi State \\ University, Box 9775, Mississippi State, MS 39762
}

Xylosandrus mutilatus is an ambrosia beetle from Asia which was first detected in Mississippi in 1999 and has now become established [1]. In China, X. mutilatus is one of the major pests attacking the trunk and branches of Chinese chestnut, Castanea mollissima Blue [2]. Timber losses in North America due to ambrosia beetle damage on an annual basis have been reported to be approximately $\$ 48.8$ million and at least 44 exotic species of Scolytinae are now established [1,3]. Unlike bark beetles that bore into the phloem and use fungi as agents to kill living trees, ambrosia beetles bore into the xylem of stressed, dying, or recently felled trees and use fungi as their primary food source [4]. Ambrosia beetles lack the enzymes capable of digesting cellulose and lignins and have developed a mutualistic association with wood-degrading fungi [5]. Ambrosia beetles have specialized structures termed mycangia, which consist of pits, sacs, pouches, tubes, or pubescent areas where fungal spores are contained and allowed to proliferate [6]. Hence, fungal spores are protected against desiccation and contamination until dispersal [4]. The mycangia can occur in numerous locations in and on beetles and all are invaginations of the cuticle. Entry and exit of spores is probably passive and enhanced by body movements [6]. The fungal spores are released from the mycangium and inoculated on the gallery walls [7]. Once established, the fungi form a symbiotic relationship with the beetle by serving as the primary food resource for larvae and adults $[6]$.

The purpose of this study was to describe the mycangium with light, scanning and transmission electron microscopy. Thirty mycangia were dissected from mature female beetles and prepared by conventional light and electron microscope methodologies. In X. mutilatus, the mycangium is found only in females and consists of a single, paired sac-like organ of ectodermal origin which lies in the forward margin of the mesonotum [4]. The inner cuticular structure of the mycangium is formed by the recurved scutellum [8]. Previous studies concerning X. mutilatus did not describe in detail the mycangium $[1,2,4,7]$. During the teneral and overwintering life stages, fungal spores have been identified from the mycangium of $X$. mutilatus as Ambrosiella, Candida, Paecilomyces, and Ceratocystis species, but Ambrosiella sp. was the only fungus present during all life stages [4].

Light microscopy images were acquired on Zeiss Axioskop 2 Light Microscope (20X and 40X objectives). Scanning microscopy images were acquired on Zeiss 360 Scanning Electron Microscope and a JEOL JSM 6500F Field Emission Scanning Electron Microscope. Transmission microscopy images were acquired on JEOL JEM 100 CX II Transmission Electron Microscope. Images obtained provide a new understanding of the morphological and taxonomical characteristic of the mesonotal mycangium of $X$. mutilatus. Future research is underway to identify the fungal species. 
[1] T. L. Schiefer and D. E. Bright, The Coleop. Bull. 58 (2004) 431-438.

[2] W. Tang, J. Zhejiang Forestry College, 17 (2000) 417-420.

[3] J. A. McLean, The Forestry Chronicle. August (1985) 295.

[4] H. Kajimura and N. Hijii, Ecol. Res. 7 (1992) 107-117.

[5] A. A. Berryman. 1989. In: Insect-Fungus interactions. N. Wilding et al., eds, Academic Press. San Diego: 145-156.

[6] R. A. Beaver. 1989. In: Insect-Fungus interactions. N. Wilding et al., eds, Academic Press.

San Diego: 121-137.

[7] H. Kajimura and N. Hijii, Entomol. Exp. Appl. 71 (1994) 121-132.

[8] G. M. Happ et al., Int. J. Insect Morphol. \& Embryol. 5 (1976) 382.

[9] Supported by a grant from US Forest Service, Contract SRS 03-CA-11330129-222
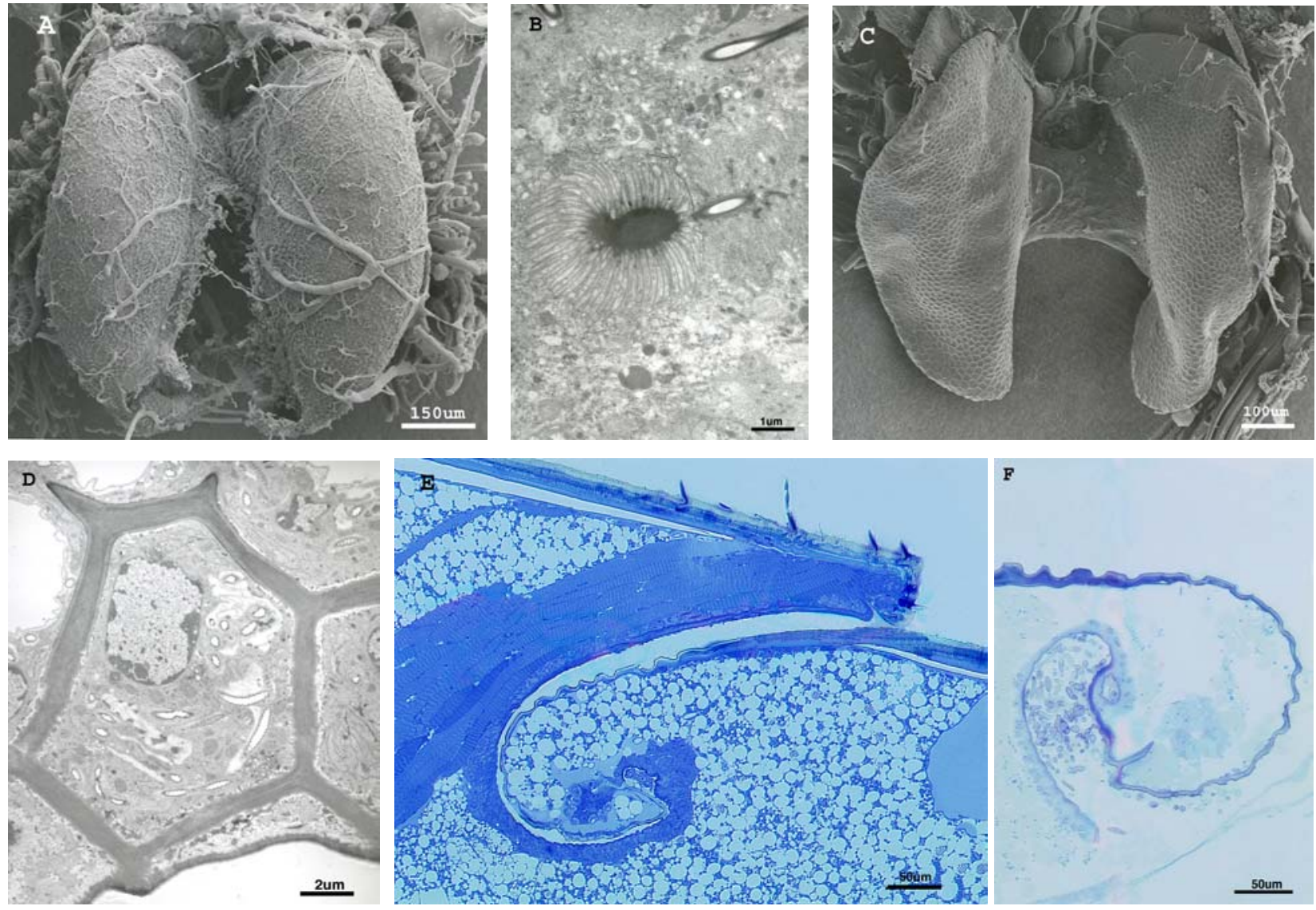

Fig. 1. Ultrastructure of the mesonotal mycangium of $X$. mutilatus (A) Outer secretory or glandular cell layer of with numerous tracheoles (B) Type-1 secretory cell found within the outer secretory layer. (C) Inner cuticular structure of the mycangium with hexagonal patterning on its surface. (D) Epithelial cell surrounded by the cuticular ridges of the inner cuticular structure. (E) Dorsal cuticular opening (formed by the recurved pronotum and scutellum) which allows entry and exit of fungal spores. (F) Fungal spores found within the mycangial bridge (area of cuticle that connects both sacs). From the mycangial bridge, fungal spores travel to each sac or exit the mycangium via the dorsal cuticular opening. 